\title{
About Ad hoc On-Demand Multi-Path Distance Vector Routing for Mobile Ad hoc Networks
}

\author{
Phu Hung Le ${ }^{1}$ \\ Hanoi University of Natural Resources and Environment ${ }^{1}$
}

\begin{abstract}
In Mobile Ad hoc NETwork (MANET), routing is one of the most important problems and is widely studied in the world. Routing greatly affects performance of the network. Multi-path routing is a technique that uses multiple paths between the source and the destination. Its advantages are that it increases throughput, reliability and reduces end-to-end delay, and so on. In this paper, we compare performance of the Ad hoc On-demand Multi-path Distance Vector routing (AOMDV) and the Ad hoc On-Demand Distance Vector Routing (AODV) in terms of Packet Delivery Fraction, Routing overhead and Nomalize Routing Load. Simulation results show that the AOMDV' packet delivery fraction outperforms that of the AODV. Our results also show that AOMDV'Routing overhead and Nomalize Routing Load are less than AODV.
\end{abstract}

Keywords: Mobile Ad Hoc Networks; Routing Protocol; AODV; AOMDV.

\section{INTRODUCTION}

A mobile ad hoc Network (MANET) is self-configuring, dynamic network in which nodes are free to move. Mobile ad hoc network has not any pre-existing communication infrastructure. Because of their unique characteristics, ad hoc networks have many applications in defense, disaster recovery, emergency services, education, healthcare, corporate conventions/meetings, indoor and personal networks, as well as sensor networks. However, there are many challenges in ad hoc networks such as limited bandwidth, low battery, high loss rate, frequent link breakage, etc., In MANET, routing protocols are divided into three categories [6]:

On-demand routing protocols only calculate a path when they need to send data. Some on-demand protocols are Ad hoc On-Demand Distance Vector Routing (AODV) [3], Dynamic Source Routing (DSR) [4], Temporally Ordered Routing Algorithm (TORA) [5].

In proactive (table-driven) protocols, the routing table and topology of network is maintained at each node. These protocols have low delay because a path to the destination is immediately available. Some famous proactive protocols are Destination-Sequenced Distance-Vector (DSDV) [2], Optimized Link State Routing (OLSR) [13].

And the third category is hybrid protocols that use both periodic and on-demand routing, for example, the Zone Routing Protocol (ZRP) [7].

Multi-path routing uses multiple paths between the source and the destination. It increases throughput, reliability and reduces end-to-end delay, and so on. Some famous Multi-path routing protocols are Ad hoc On-demand Multi-path Distance Vector routing (AOMDV) [1], Split Multipath Routing (SMR)[12].

In this paper, we analyze performance of the AOMDV.

This paper is organized as follows. Section II and III introduce the detail structure of AODV and AOMDV. In section IV, we compare the AOMDV to the AODV and conclusion in section V.

\section{AD HOC ON-DEMAND DISTANCE VECTOR ROUTING}

Ad hoc On-Demand Distance Vector Routing (AODV) [3,6] is also another typical reactive protocol. Different from DSR, AODV conducts a broadcast route discovery mechanism to find the route. To maintain the most recent routing information between nodes, AODV uses the concept of destination sequence numbers.

A. Path Discovery

When a source node needs to transmit packets to another node for which it has no routing information in its table, the Path Discovery process is initiated. All nodes maintain two separate counters: a node sequence number and a broadcast_id.

The source node discovers the path by broadcasting a route request (RREQ) packets to its neighbors.

The fields in RREQ include:

$<$ source_addr, source_sequence_\# broadcast_id, dest_addr, dest_sequence_\#, hop_cnt $>$

The pair < source_addr, broadcast_id > uniquely specifies a RREQ. When the source sends a new RREQ, broadcast_id increases. If each neighbor satisfies the RREQ, it will sends a route reply (RREP) back to the source, or rebroadcasts the RREQ to its own neighbors after increasing the hop_cnt. 


\section{International Journal of Advanced Research in Computer and Communication Engineering}

Vol. 9, Issue 10, October 2020

DOI 10.17148/IJARCCE.2020.91001

When an intermediate node receives a RREQ with the same broadcast_id and source address that it has already received a RREQ, it deletes the redundant RREQ and does not rebroadcast. If a node cannot satisfy the RREQ, it saves the information below for implementation of the reverse path setup, as well as the forward path setup that will accompany the transmission of the eventual RREP:

Destination IP address

Source IP address

Expiration time for reverse path route entry

Source node sequence number

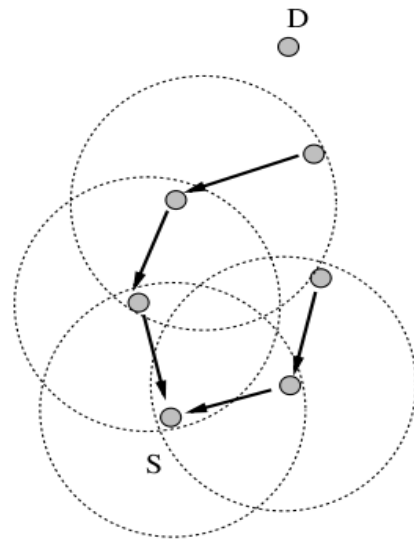

Figure 1. Reverse Path Formation

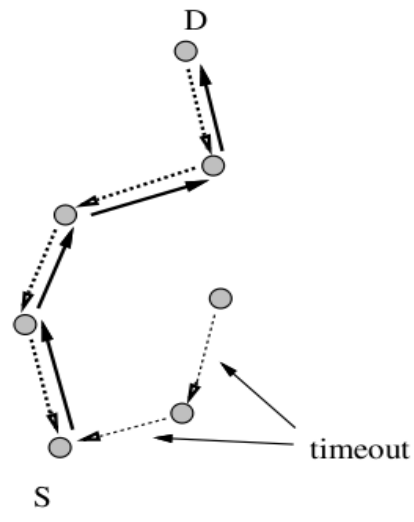

Figure 2. Forward Path Formation

Figure 3.5: Reverse and forward in AODV protocol

\section{B. Reverse path setup}

A RREQ consists of two sequence numbers: the source sequence number and the destination sequence number. The source sequence number keeps freshness information about reverse route to source. The destination sequence defines how fresh the route to the destination must be before it can be accepted by the source. To form a reverse path, a node keeps the address of the neighbor from which it received the first copy of the RREQ.

\section{Forward Path Setup}

When a RREQ arrives at a node that has a current route to the destination, first, the receiving node checks that the RREQ was received over a bi-directional link. If an intermediate node has a route entry for the desired destination, it compares the destination sequence number in its own route entry to the destination sequence number in the RREQ. The intermediate node can reply if it has a route with a sequence number that is higher than or equal to that contained in the RREQ. On the contrary, if the RREQ's sequence number for the destination is higher than that recorded by the intermediate node, the intermediate node must rebroadcast the RREQ. If it has a current route to the destination, and if the RREQ has not been processed previously, the node then sends a route reply packet (RREP) back to its neighbor from which it received the RREQ. A RREP consists of the following information: < source_addr, dest_addr, dest_sequence_\#, hop_cnt, lifetime $>$

\section{Path Maintenance}

A node that does not belong to an active path moves then it does not affect the routing to that path's destination. If the movement of a source node occurs during an active session, it can reinitiate the route discovery procedure to find a new route to the destination. When the destination or some intermediate node moves, a special RREP is sent to the affected source nodes. HELLO messages can be periodically sent to ensure symmetric links, as well as to detect link failures. A link failure is also known if a packet cannot be successfully forwarded to the next hop. Once the next hop cannot be reached, the node upstream of the break sends an unsolicited RREP with a fresh sequence number (i.e., a sequence number that is one greater than the previously known sequence number) and hop count of 1 to all active upstream neighbors. Then, those nodes relay that message to their active neighbors and so on. This process continues until all active source nodes are notified.

\section{AD HOC ON-DEMAND MULTI-PATH DISTANCE VECTOR ROUTING}

Ad hoc On-demand Multi-path Distance Vector routing (AOMDV) [1] is a multi-path extension to a well-studied single path routing protocol known as Ad hoc On-demand Distance Vector (AODV). It is a protocol with loop freedom and uses alternate disjoint paths. AOMDV has several same characteristics as AODV. They are on demand protocol and use the 


\section{International Journal of Advanced Research in Computer and Communication Engineering}

Vol. 9, Issue 10, October 2020

\section{DOI 10.17148/IJARCCE.2020.91001}

distance vector concept and hop-by-hop routing approach. The main difference is the number of routes found in each route discovery.

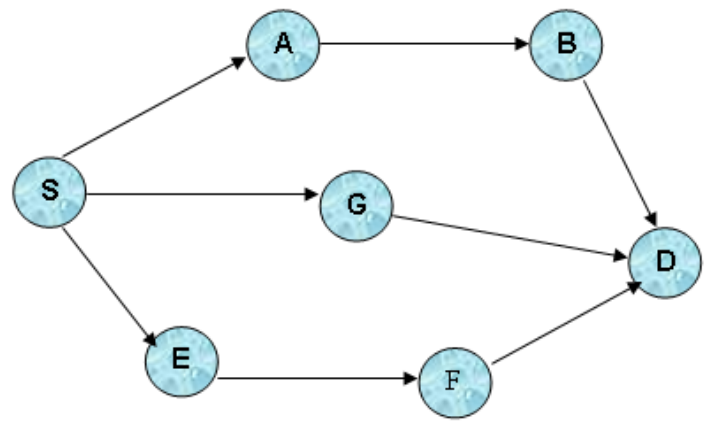

Figure 3.7: Paths in AOMDV protocol

In AOMDV, a RREQ packet is sent from the source to the destination establishing multiple reverse paths both at intermediate nodes and the destination. Multiple RREPs come back from these reverse paths to form multiple forward paths to the destination at the source and intermediate nodes. AOMDV also provides intermediate nodes with alternate paths as they are found to be useful in reducing route discovery frequency.

The core of the AOMDV protocol is that it efficiently finds multiple loop-free and disjoint paths by using a flood-based route discovery. AOMDV route update rules, applied locally at each node, have an important role in maintaining loopfreedom and disjointness properties. AOMDV is based as much as possible on the routing information already available in the AODV protocol, thereby limiting the overhead suffered from discovering multiple paths. AOMDV does not conduct any special control packets. In fact, a few extra fields in routing control packets (i.e., RREQs, RREPs, and RERRs) as well as extra RREPs and RERRs for multipath discovery and maintenance create the only additional overhead in AOMDV relative to AODV. AOMDV uses destination sequence number to maintain a loop freedom.

\section{IV.PERFORMANCE EVALUATION}

A. Simulation environment: We experiment with 50 nodes moving within an area of $550 \mathrm{~m} \times 550 \mathrm{~m}$. Protocol is implanted in NS-2 with 11Mbps 802.11 channels. The traffic source is Constant Bit Rate (CBR). The distributed coordination function (DCF) of IEEE 802.11 for wireless LANs is used as the MAC layer. The Random Waypoint and Two-Ray Ground models have been used as propagation model and mobility model, respectively.

B. Simulation results: In the simulations, we compare the performance between AOMDV and AODV for:

1-Packet delivery fraction (PDF)

2- Routing overhead

3-Nomalize Routing Load (NRL)

As shown in Figure 4, the PDF of AOMDV outperforms that of AODV with 10, 15 and 20 connections.

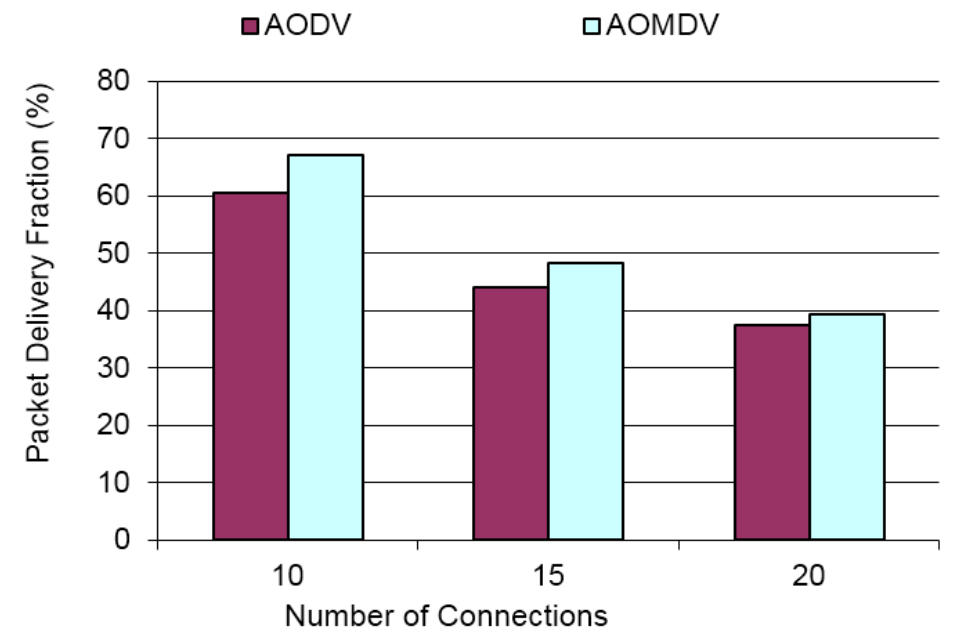

Figure 4: Packet delivery fraction 


\section{International Journal of Advanced Research in Computer and Communication Engineering}

Vol. 9, Issue 10, October 2020

DOI 10.17148/IJARCCE.2020.91001

We can see in Figure 5 that Routing overhead of two protocols increases when the number of connections increases. However, Routing overhead of AOMDV is less than that of AODV.

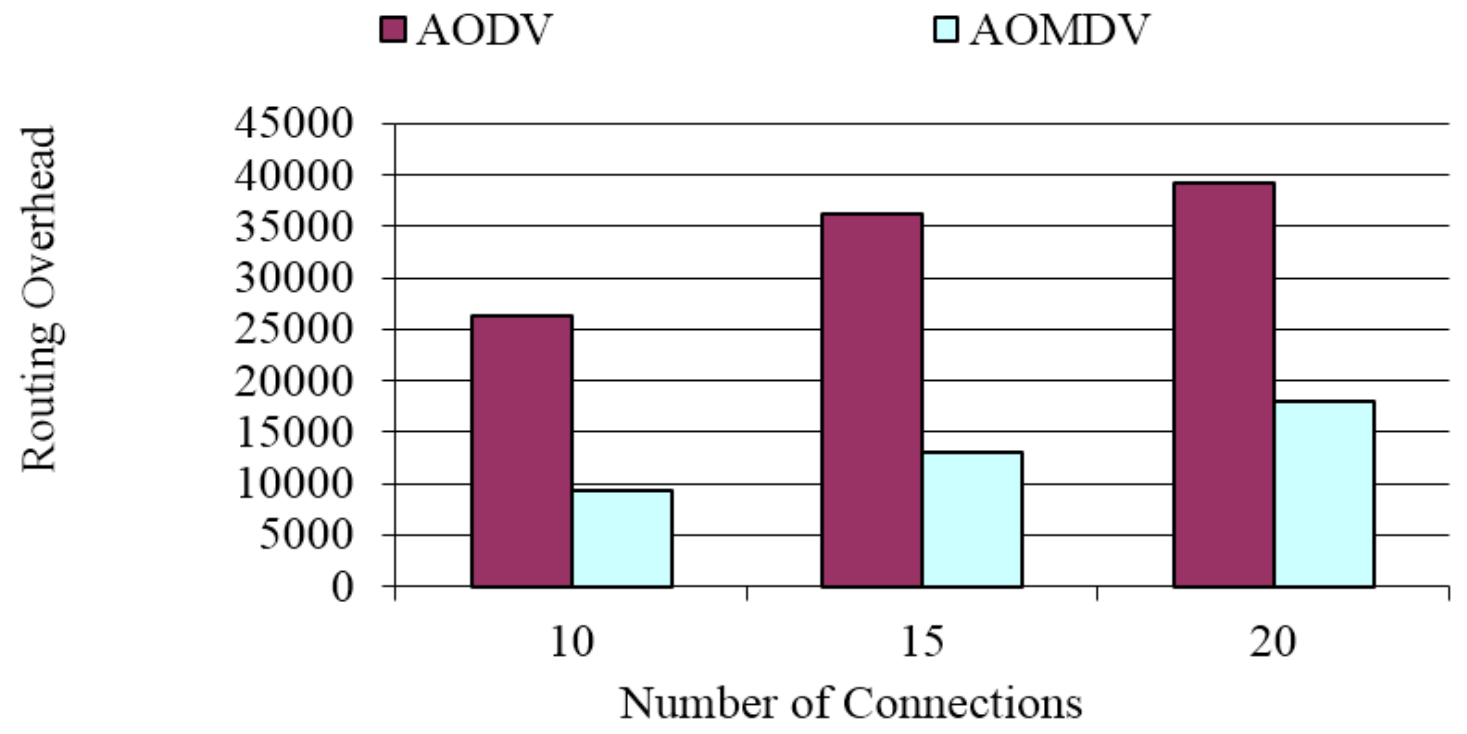

Figure 5: Routing overhead

When the number of connections rises Nomalize Routing Load of two protocols increase significantly. Nomalize Routing Load of AOMDV is much lower than that of the AODV as shown in Figure 6.

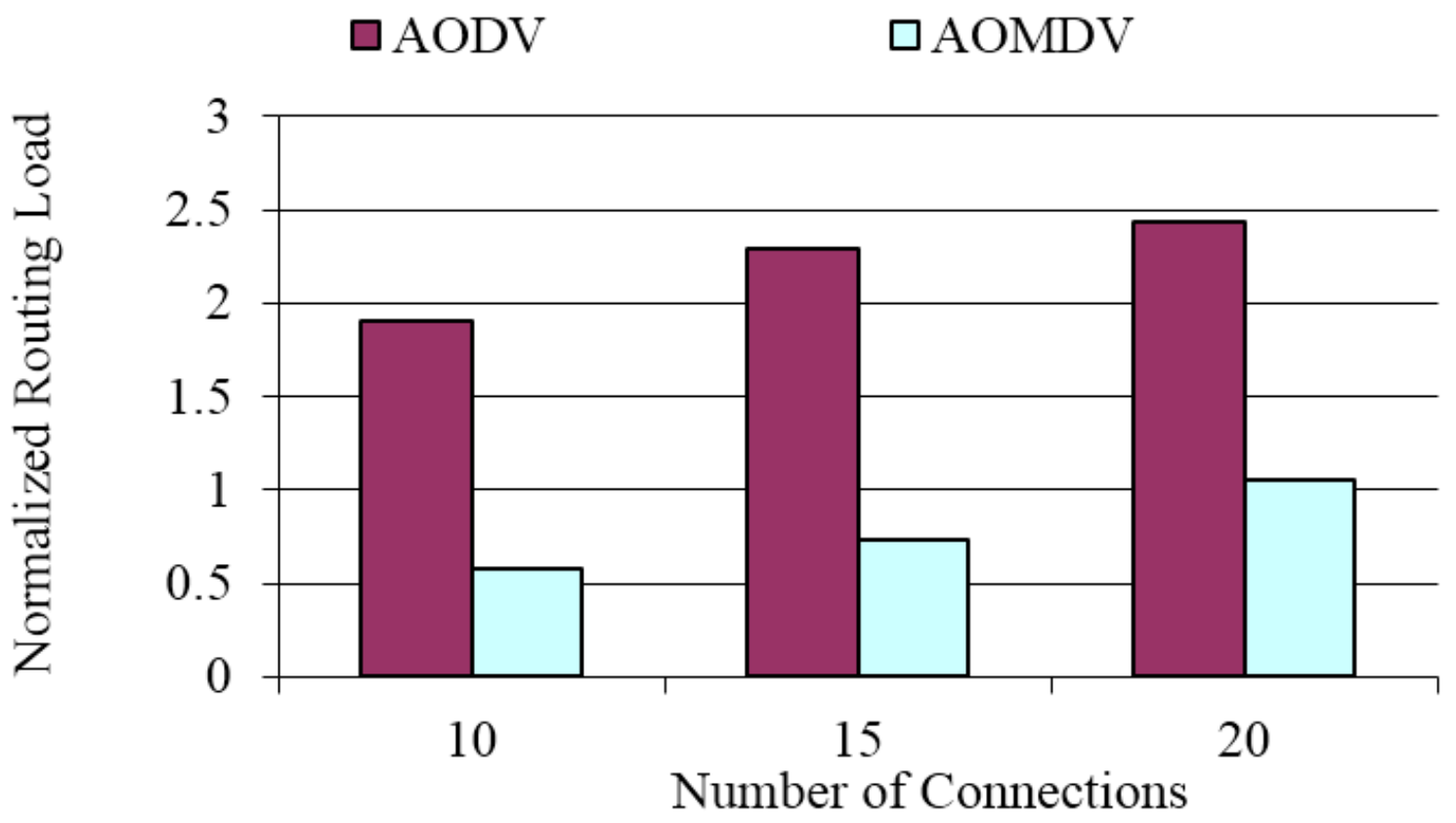

Figure 6: Nomalize Routing Load

\section{CONCLUSION}

In this paper, we analyze perfomrmance of the Ad hoc On-demand Multi-path Distance Vector routing (AOMDV) by comparing with Ad hoc On-Demand Distance Vector Routing (AODV). We can see that in term of the Packet delivery fraction, AOMDV is better than AODV. In term of the Routing overhead and Nomalize Routing Load, AOMDV is less than AODV. 


\section{International Journal of Advanced Research in Computer and Communication Engineering}

Vol. 9, Issue 10, October 2020

DOI 10.17148/IJARCCE.2020.91001

\section{REFERENCES}

[1] Marina, M. K. and Das, S. R., "On-demand Multipath Distance Vector Routing for Ad Hoc Networks", Wireless communications and mobile computing, 2005.

[2] Perkins CE, Bhagwat P. Highly dynamic destination-sequenced distance-vector routing (DSDV) for mobile computers. In Proceedings of ACM Sigcomm, 1994.

[3] C. E. Perkins and E. M. Royer. Ad Hoc On Demand Distance Vector (AODV) Routing. draft-ietf-MANET-aodv-02.txt, Nov. 1998. (work in progress).

[4] David B. Johnson, David A. Maltz, Josh Broch, “DSR: The Dynamic Source Routing Protocol for Multi-Hop Wireless Ad Hoc Networks,” Ad Hoc Networking, Addison-Wesley, 2001:139 172.

[5] Park and S. Corson, "Temporally-ordered routing algorithm (TORA) version 1 functional specification", IETF Draft: draft-ietf-MANET-tora-spec04.txt, 2001.

[6] Phu Hung Le, "Performance Comparison of the Ad hoc On-Demand Distance Vector Routing Protocol and The Dynamic Source Routing Protocol for Mobile Ad hoc Networks", IJARCCE, 2019

[7] Haas and Pearlman Zone Routing Protocol,1997.

[8] Phu Hung Le, Guy Pujolle, Thi-Mai-Trang Nguyen, "An Interference-aware multi-path routing protocol for Mobile Ad hoc Network", proc of ICNSC 2011, Delft, the Netherlands, April 2011, IEEEXplore, pages 503 - 507.

[9] Phu Hung Le, Guy Pujolle, "A Link-disjoint interference-aware multi-path routing protocol for Mobile Ad hoc Network", proc of The International Conference on Digital Information and Communication Technology and its Applications(DICTAP 2011), Dijon, France, June 2011, Springer LNCS, pages 649-661.

[10] Phu Hung Le, "A Performance Evaluation of Multi-Path Routing Protocols for Mobile Ad Hoc Network", the 10th IEEE/IFIP International Conference on Embedded and Ubiquitous Computing, Paphos, Cyprus, December 2012

[11] Phu Hung Le "A New Efficient Multi-path Routing Protocol for Mobile Ad hoc Networks", International Journal of Advanced Research in Computer and Communication Engineering, 2017.

[12] S J Lee, M Gerla, "Split MultiPath Routing with Maximally Disjoint Paths in Ad Hoc Networks", proc of IEEE ICC 2001

[13] T. Clausen, P. Jacquet, IETF Request for Comments: 3626, Optimized Link State Routing Protocol OLSR, October 2003.

\section{BIOGRAPHY}

Phu Hung Le received the Ph.D degree in Computer Science from Pierre and Marie Curie University (Paris 6 University), France. Phu Hung Le is the author of many articles and is a reviewer for many international conferences and journals. His research interests are Mobile Ad hoc Networks and Sensor Networks. 